\title{
The genesis solar wind concentrator target Mass fractionation characterised by neon isotopes
}

\section{Conference Paper}

Author(s):

Heber, V. S.; Wiens, R. C.; Reisenfeld, D. B.; Allton, J. H.; Baur, H.; Burnett, D. S.; Olinger, C. T.; Wiechert, U.; Wieler, R.

Publication date:

2007

Permanent link:

https://doi.org/10.3929/ethz-b-000005276

Rights / license:

In Copyright - Non-Commercial Use Permitted

Originally published in:

Space Science Reviews 130(1-4), https://doi.org/10.1007/s11214-007-9179-1 


\title{
The Genesis Solar Wind Concentrator Target: Mass Fractionation Characterised by Neon Isotopes
}

\author{
V.S. Heber · R.C. Wiens • D.B. Reisenfeld • J.H. Allton • \\ H. Baur · D.S. Burnett · C.T. Olinger · U. Wiechert • \\ R. Wieler
}

Received: 22 December 2006 / Accepted: 26 March 2007 /

Published online: 15 May 2007

(C) Springer Science+Business Media, Inc. 2007

\begin{abstract}
The concentrator on Genesis provided samples of increased fluences of solar wind ions for precise determination of the oxygen isotopic composition. The concentration process caused mass fractionation as a function of the radial target position. This fractionation was measured using Ne released by UV laser ablation and compared with modelled $\mathrm{Ne}$ data, obtained from ion-trajectory simulations. Measured data show that the concentrator performed as expected and indicate a radially symmetric concentration process. Measured concentration factors are up to $\sim 30$ at the target centre. The total range of isotopic fractionation along the target radius is $3.8 \%$ /amu, with monotonically decreasing ${ }^{20} \mathrm{Ne} /{ }^{22} \mathrm{Ne}$ towards the centre, which differs from model predictions. We discuss potential reasons and propose future attempts to overcome these disagreements.
\end{abstract}

Keywords Solar wind · Noble gases · Genesis oxygen isotopic analysis · UV laser ablation

\author{
V.S. Heber $(\varangle) \cdot$ H. Baur $\cdot$ R. Wieler \\ Isotope Geology and Mineral Resources, ETH, 8092 Zürich, Switzerland \\ e-mail: heber@erdw.ethz.ch \\ R.C. Wiens - C.T. Olinger \\ LANL, Space \& Atmospheric Science, Los Alamos, NM 87544, USA \\ D.B. Reisenfeld \\ Physics and Astronomy, University of Montana, Missoula, MT 59812, USA \\ J.H. Allton \\ JSC, 2101 NASA Parkway, Houston, TX 77058, USA \\ D.S. Burnett \\ CalTech, JPL, Pasadena, CA 91109, USA \\ U. Wiechert \\ AG Geochemie, FU Berlin, 12249 Berlin, Germany
}




\section{The Genesis Solar Wind Concentrator}

The solar wind concentrator on the Genesis spacecraft was designed to provide a sample of increased concentration of solar wind atoms to allow high precision laboratory analysis of the isotopic composition of light elements (Burnett et al. 2003; Nordholt et al. 2003; Wiens et al. 2003), particularly of oxygen and nitrogen. The solar wind is a proxy for the composition of the sun and the primordial solar nebula. The oxygen isotope abundances in the solar wind are therefore fundamental to understand the observed differences in the oxygen isotopic composition between different solar system bodies and different constituents of primitive meteorites (Clayton 2003; Wiens et al. 2004; Hashizume and Chaussidon 2005).

The concentrator was an electrostatic mirror with targets placed at the focal point, designed to concentrate the fluence by about a factor of 20 on average (Wiens et al. 2003). A hydrogen rejection grid prevented the targets from radiation damage during exposure. Incoming ions with mass/charge ratios of 2.0-3.6 (masses 4 to $28 \mathrm{amu}$ ) were accelerated to increase implantation depths and therefore reduce backscatter losses. The ions were reflected and focused onto the target by a domed grid and a mirror (see Wiens et al. 2003 and Nordholt et al. 2003 for a complete description). This concentration process resulted in an unavoidable instrumental mass fractionation varying as function of the radial target position. The performance of the concentrator was tested on prototypes in an ion beam facility prior to launch. However, the tests could not perfectly simulate the solar wind in terms of charge state-, angular-, and velocity distributions. Instead, an ion trajectory simulation code SIMION 7.0, validated with some ion beam testing, was used to simulate the concentrator performance under predicted solar wind conditions (Wiens et al. 2003). After return of the spacecraft, these simulations were repeated using solar wind conditions encountered during the mission.

The aim of this work is to verify the post-flight simulation by direct measurement of the mass fractionation on the concentrator target as function of the radial position. To this end, we analysed the $\mathrm{Ne}$ abundances and isotopic composition along 2 of the 4 arms of the "gold cross" of the concentrator target at high spatial resolution (Fig. 1). Major attention was paid to achieve high precision $\mathrm{Ne}$ isotope data of the same order as the intended accuracy of oxygen analyses of $\sim 0.1 \%$. Neon is especially suitable since it is (i) abundant in the solar wind, therefore not influenced by terrestrial contamination, (ii) similar in mass to oxygen and nitrogen, and (iii) its bulk solar wind composition is well known

Fig. 1 The Genesis concentrator target, shortly after return (note the large dust grain on arm 3). The concentrator target consists of four sub-targets mounted by the gold cross onto the base plate. The cross was cut for the $\mathrm{Ne}$ analyses and results are presented for the arms 12 and 9. ( $\alpha-\mathrm{C}:{ }^{13} \mathrm{C}$ diamond film on substrate, DOS: diamond-like carbon on $\mathrm{Si}$ )

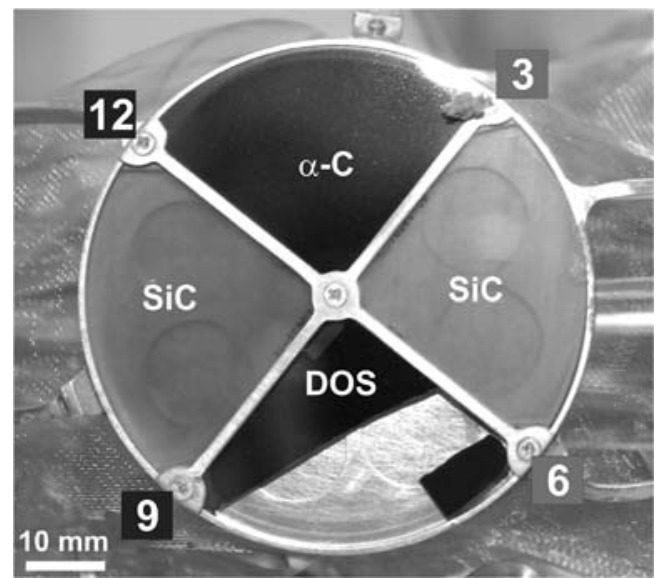


from many investigations (e.g., Apollo Solar Wind Composition Experiment (e.g., Geiss et al. 2004); lunar soils; in situ-analyses, e.g., CELIAS/SOHO (e.g., Kallenbach et al. 1997); and preliminary results from passive collectors from Genesis (Heber et al. 2007; Mabry et al. 2007). Here we report our data and compare experimentally deduced concentration factors and mass fractionation with the post-flight simulation.

\section{Experimental}

\subsection{The Simulation}

The performance of the concentrator, i.e. the process of solar wind ion implantation into the concentrator target, was numerically simulated before launch of the Genesis spacecraft. The simulation was re-run at return using the actual solar wind parameters and spacecraft working conditions encountered during the collection period. The ion simulation code used as well as the influence of all parameters included in the model are described in detail by Wiens et al. (2003). Briefly, the simulation determines the electrostatic potential of points in free space and then determines the trajectories of ions through space from a given input velocity, angle, mass, and charge state. The spacing of the computational nodes was $0.67 \mathrm{~mm}$, limited by the software. Monte Carlo routines were used to give each ion's input position at a plane just above the concentrator, the initial angle, and charge state. Different ion velocities were simulated using separate simulations, as the concentrator voltages had to be adjusted for each velocity in simulation as in reality. Five different simulations are used to cover the velocity distribution using equally spaced bins. For most simulations at least one million ions are flown. The output of the simulation was a distribution of ions along radial positions on the target for each solar wind speed, which were averaged over $5 \mathrm{~mm}$ bins to improve statistics. Final ${ }^{20} \mathrm{Ne}$ and ${ }^{22} \mathrm{Ne}$ concentrations and the corresponding isotopic fractionation factors are averages weighted by the respective fluences per bin based on the proton velocity distribution with a correction to account for the velocity difference between heavy ions and protons.

The parameters influencing the distribution of implanted isotopes into the concentrator target are the charge state-, the velocity-, and the angular distributions of the incoming solar wind ions as well as the ion loss due to backscattering. The charge state distribution of $\mathrm{Ne}$ and $\mathrm{O}$ during the Genesis collection period was contemporarily measured by the SWICS instrument on board the Advanced Composition Explorer (ACE) (Gloeckler et al. 1998). The Genesis Ion Monitor continuously recorded the proton velocity (Barraclough et al. 2003). The angular distribution resulted from the intrinsic angular distribution of the solar wind as well as the instrument orientation, including spacecraft motion, pointing, nutation, and wobble, and instrument-to-instrument alignment. The Genesis spin axis was nominally pointed 4.5 degrees ahead of the sun, which is the apparent average incoming direction of the solar wind when convolved with spacecraft motion around the sun. Daily correction manoeuvres were done to keep the spacecraft nominally within 0.5 degrees of this position, not counting nutation and wobble, as it orbited the Sun. The backscatter losses of Ne isotopes implanted into Au were calculated using the SRIM code (Ziegler 2004), taking into account the measured solar wind velocity distribution and SIMION-modelled angles of incidence on the target. Backscatter losses are included in the simulated data. Model data are shown in Fig. 2. Error bars of modelled $\delta^{22} \mathrm{Ne}$ reflect $1-\sigma$ statistical uncertainties. Note that the modelled $\mathrm{Ne}$ abundances and isotopic composition are directly comparable to the actual measured $\mathrm{Ne}$ data. 
Fig. 2 Neon concentrations (upper panel) and isotopic composition (lower panel) of arms 12 (black circles, black fit line) and 9 (grey triangles, grey fit line). Data have been fitted by sigmoid functions (error weighted for concentrations, unweighted for the $\delta^{22} \mathrm{Ne} ; 95 \%$ confidence bands for the fits are given). The innermost 2 data points of arm 9 are not considered (see text). Also shown are modelled data (vertical dashes connected by a black line). All data are plotted as function of the distance from the centre of the concentrator target. Uncertainties are given as $2-\sigma$ errors of the mean for the measured and as 1- $\sigma$ statistical errors for the modelled data



\subsection{Measurement of Neon in the Concentrator Target}

Neon concentration and isotope composition were analysed on the "gold cross" used to mount the individual concentrator targets onto a base plate. This cross consists of stainless steel coated with electroplated $\mathrm{Au}(0.5-1 \mu \mathrm{m}$ thick). For analysis the cross was cut in order to allow single arms to be analysed separately. Here we present the results obtained from two arms, 9 (NASA code: 60009.2) and 12 (60009.1) (Fig. 1). Both arms were free of visible contamination and "hard landing"-induced damages, except for a few scratches at the outer part of arm 9.

Neon was released from single spots $(\sim 100 \mu \mathrm{m}$ in diameter $)$ ablated by an UV laser ( $\lambda=248 \mathrm{~nm}$ : arm $12 ; \lambda=213 \mathrm{~nm}$ : arm 9). Pit depth was a few microns, considerably larger than the maximum implantation depth of solar wind $\mathrm{Ne}$ in the concentrator targets of $\sim 250 \mathrm{~nm}\left({ }^{22} \mathrm{Ne}^{8+}\right.$ at $800 \mathrm{~km} / \mathrm{s}$ vertically implanted into Au; SRIM code, Ziegler 2004). Both arms were extensively sampled at 12 positions about equally distributed along the whole arm. Several measurements were done at each radial position. We reject the two innermost samples of arm 9 (shown in brackets in Fig. 2) since we cannot exclude incomplete ablation caused by partly shadowing of the UV beam at the edge using the $213 \mathrm{~nm}$ laser, which has a shorter focal distance than the $248 \mathrm{~nm}$ laser.

Neon was analysed with a very sensitive mass spectrometer (Baur 1999). All samples were corrected by a blank carried out on flight-spare material using identical ablation con- 
ditions. However, blank contributions were insignificant. In Fig. 2 the ${ }^{20} \mathrm{Ne}$ concentrations and the $\mathrm{Ne}$ isotopic composition are presented as mean values at each radial position. $\mathrm{Re}-$ producibilities are calculated as $2-\sigma$ error of the mean. For the ${ }^{20} \mathrm{Ne} /{ }^{22} \mathrm{Ne}$ ratio these uncertainties vary between $0.2-5 \%$ and are on average $1.8 \%$. Thus, the uncertainties of the measured $\mathrm{Ne}$ isotope ratios are in the range of the intended accuracy of the oxygen isotope analyses.

\section{Comparison of Measured and Modelled Data}

\subsection{Neon Concentrations}

The measured ${ }^{20} \mathrm{Ne}$ concentrations of both gold cross arms agree within their $95 \%$ confidence level along the entire radius of the concentrator target, except for a 5\% difference at the inner part of the arms (Fig. 2). This suggests, at least for the quarter of the concentrator target considered here, that the concentration process was radially symmetric. The modelled ${ }^{20} \mathrm{Ne}$ concentrations are based on concentration factors obtained from the simulation. These concentration factors were multiplied by the ${ }^{20} \mathrm{Ne}$ fluence of $(1.22 \pm 0.01) \times 10^{12}$ atoms $/ \mathrm{cm}^{2}$ measured in a DOS (diamond-like carbon on $\mathrm{Si}$ ) target from a passive Genesis collector sampling the bulk solar wind (Heber et al. 2007) that is corrected for the somewhat shorter exposure time of the concentrator target relative to the bulk collector. Carbon is a most suitable target to determine solar wind Ne fluences and isotopic composition. Its atomic mass, which is lower than that of $\mathrm{Ne}$, minimized loss and fractionation of $\mathrm{Ne}$ as a consequence of backscattering. Measured and modelled data both show monotonically increasing ${ }^{20} \mathrm{Ne}$ concentrations from the edge to the centre of the concentrator target by about a factor of 10 . It is remarkable that the relative increase of the ${ }^{20} \mathrm{Ne}$ concentration is similar in the measured and modelled data, however, both differ in their absolute concentrations. According to the simulation, concentration factors higher than 40 were expected at the centre of the target, whereas the measured data revealed maximum concentration factors of only 30 . Measured ${ }^{20} \mathrm{Ne}$ concentrations are thus about $30 \%$ lower than modelled values. Uncertainties in the calibration of the mass spectrometer as the cause of this difference can be ruled out since these would have cancelled out when relating the measured Ne data in Au to those in DOS. Furthermore, various independent noble gas calibration reservoirs in our laboratory agree to within a few percent.

\subsection{Neon Isotopic Composition}

The implanted solar Ne isotopes are fractionated as function of the radial position on the concentrator target, as was expected according to pre-flight numerical simulations of the implantation process (Wiens et al. 2003). At the edge of the concentrator target, $\mathrm{Ne}$ is slightly enriched in the light isotope $\left({ }^{20} \mathrm{Ne} /{ }^{22} \mathrm{Ne}\right.$ : 14.05) relative to bulk solar wind $\mathrm{Ne}$. Towards the centre, Ne monotonically becomes heavier with a ${ }^{20} \mathrm{Ne} /{ }^{22} \mathrm{Ne}$ ratio of $\sim 13.04$ at the centre of the concentrator target. To compare with modelled data, the Ne isotopic composition is expressed as the permil deviation of the measured ${ }^{22} \mathrm{Ne} /{ }^{20} \mathrm{Ne}$ from a standard value in Fig. 2. As a standard we used our bulk solar wind ${ }^{22} \mathrm{Ne} /{ }^{20} \mathrm{Ne}$ of $0.0724 \pm 0.0001$ measured in a DOS target of a bulk passive collector (Heber et al. 2007). The uncertainty of this value is not included in the $\delta^{22} \mathrm{Ne}$ in Fig. 2. The DOS target is expected to cause negligible Ne isotope fractionation due to backscattering. The concentrator was exposed for almost the same period (803.28 days) as the passive collectors sampling bulk solar wind (852.83 days), apart 
from those $6 \%$ of the total time when the concentrator was turned to a stand-by mode. This was during spacecraft manoeuvres, maintenance work on the $\mathrm{H}$ rejection grid and periods with solar wind velocities persistently above $800 \mathrm{~km} / \mathrm{s}$ to prevent additional mass fractionation when heavier particles would preferentially hit the mirror. Since these periods contributed $<0.2 \%$ to the total proton fluence and the intervals the concentrator was turned off for maintenance work were not biased to any particular solar wind condition, we can safely assume that the Ne collected by the DOS target on the bulk passive collector well represents the true isotopic composition of the Ne intercepting the concentrator. Note that using a lower or higher standard value would shift the fractionation curve along the $y$-axis without changing the total extent of fractionation.

The isotope fractionation curves obtained from both arms of the gold cross are indistinguishable within their uncertainties. This again shows radially symmetric operation of the concentrator. For comparison, the $\delta^{22} \mathrm{Ne}$ obtained from the simulation is also shown in Fig. 2. At the high concentration side of the target $(0-17 \mathrm{~mm})$ the measured and modelled curves have similar slopes but are offset relative to each other. They both show a heavier $\mathrm{Ne}$ isotopic composition towards the centre of the target, but the measured absolute fractionation (maximum $\delta^{22} \mathrm{Ne}:+60 \%$ ) is larger than the modelled one (maximum $\delta^{22} \mathrm{Ne}:+40 \%$ ). However, at the lower concentration side $(17-31 \mathrm{~mm})$, measured and modelled data show very different trends. Whereas the measured $\delta^{22} \mathrm{Ne}$ continuously decreases towards the edge, the modelled curve attains a minimum around $20 \mathrm{~mm}$. Thus, the measured target-wide instrumental mass fractionation along the radius is considerably larger (3.8\%/amu) than the modelled one $(2.1 \% / \mathrm{amu})$.

\section{Consequences for the Correction of Oxygen Isotope Data from the Concentrator Target}

The Ne data here are basically very encouraging, as they prove that the concentrator performed essentially as expected. The concentrator targets therefore will serve their intended purpose well. However, the differences between measured and modelled Ne concentrations and-especially-isotopic compositions need to be understood for a proper mass fractionation correction of the eventual oxygen data. We are currently exploring why simulated and measured data differ more than expected. In pre-flight comparisons of ion beam tests and SIMION modelling, it was recognized that the model tended to predict ion impact positions somewhat closer to the centre of the target than were observed, and it appears that post-flight results are consistent with this. The reason for the discrepancy was never understood. One concern was the spatial resolution of the model. At $0.67 \mathrm{~mm}$ per grid unit, critical features such as the ion trajectories at the locations where they were turned around in the mirror section may have been compromised. A newer version of the software is now being released which may allow an improvement in the modelling. A comparison between pre- and post-flight simulations (compare Fig. 2 with the equivalent figure in Wiens et al. 2003) showed that the isotopic composition as function of the radial target position is very sensitive to solar wind conditions and other model parameters. One important difference to pre-flight assumptions was the actual solar wind velocity distribution, which shifted to higher values. This resulted on the one hand in somewhat lower concentration factors (because faster ions are more difficult to focus), and on the other hand in a preferred concentration of heavier isotopes toward the centre. However, including these new parameter values in the post-flight simulations only partially improved the agreement with the measured data. 
One concern prior to launch has been that the concentrator might become misaligned during operation, leading to a somewhat different behaviour than modelled. Unfortunately, the hard landing prevented remapping the concentrator. Therefore, we have to assume that grid shapes remained unchanged. The very similar data obtained on two different arms of the gold cross are strongly encouraging that this assumption is justified. Major misalignments of the concentrator can therefore very likely be excluded as reason for the differences between measured and modelled $\mathrm{Ne}$ data. Future data from the two remaining arms are expected to corroborate this conclusion.

A potentially major problem may be the substantial backscatter loss of $\mathrm{Ne}$ from the high atomic mass element $\mathrm{Au}$, which amounts to $30-40 \%$ for the prevailing range of angles of incidence of $40-60^{\circ}$. The respective correction was based on SRIM simulations (Ziegler 2004), taking into account the variable angle of incidence of the ions. However, the actual gold cross has a quite rough surface, which might have resulted in even larger backscatter losses than predicted. We will study this possibility by implantation experiments carried out on identical spare flight materials and variable angles of incidence of the ion beam to imitate the irradiation of the concentrator by the solar wind. Depending on the outcome of these experiments, it may be strongly advisable also to analyse Ne in some of the concentrator targets themselves. These materials have a much lower atomic mass than gold and hence require much lower backscatter loss corrections. Thus, minor differences between losses of oxygen and $\mathrm{Ne}$ and the corresponding differences in isotopic fractionation of the two elements should accurately be predictable by SRIM simulations. Note that a single $\mathrm{Ne}$ analysis on a concentrator target would only consume $\sim 0.005 \mathrm{~mm}^{2}$ of target area.

In summary, our data show that the Genesis solar wind concentrator worked essentially as designed. Experimentally determined Ne concentrations and isotopic composition as function of the radial position at the concentrator target support a radially symmetric operation of the concentrator. Future work will concentrate on the understanding of the fractionation to eliminate the remaining differences in abundances and isotopic composition between measured and modelled data.

Acknowledgements We acknowledge the financial support by the Swiss National Science Foundation. We thank for the support by the NASA Discovery Mission Office.

\section{References}

B.L. Barraclough, E.E. Dors, R.A. Abeyta, J.F. Alexander, F.P. Ameduri, J.R. Baldonado, S.J. Bame et al., Space Sci. Rev. 105, 627-660 (2003)

H. Baur, EOS Trans. Suppl. 46, F1118 (1999)

D.S. Burnett, B.L. Barraclough, R. Bennett, M. Neugebauer, L.P. Oldham, C.N. Sasaki, D. Sevilla et al., Space Sci. Rev. 105, 509-534 (2003)

R.N. Clayton, in Treatise in Geochemistry, ed. by A.M. Davis, vol. 1 (2003), pp. 129-142

J. Geiss, F. Bühler, H. Cerutti, P. Eberhardt, C.H. Filleux, J. Meister, P. Signer, Space Sci. Rev. 110, 307-335 (2004)

G. Gloeckler, J. Cain, F.M. Ipavich, E.O. Turns, P. Bedini, L.A. Fisk, T.H. Zurbuchen et al., Space Sci. Rev. 86, 497-539 (1998)

K. Hashizume, M. Chaussidon, Nature 434, 619-622 (2005)

V.S. Heber, H. Baur, D.S. Burnett, R. Wieler, Helium and neon isotopic and elemental composition in different solar wind regime targets from the Genesis mission. Lunar Planet. Sci. Conf. 38th, abstract CD \#1894 (2007)

R. Kallenbach, F.M. Ipavich, P. Bochsler, S. Hefti, D. Hovestadt, H. Grünwaldt, M. Hilchenbach et al., J. Geophys. Res. 102, 26895-26904 (1997)

J.C. Mabry, A.P. Meshik, C.M. Hohenberg, Y. Marrocchi, O.V. Pravdivtseva, R.C. Wiens, C. Olinger et al., Refinement and implications of noble gas measurements from Genesis. Lunar Planet. Sci. Conf. 38th, abstract CD \#2412 (2007) 
J.E. Nordholt, R.C. Wiens, R.A. Abeyta, J.R. Baldonado, D.S. Burnett, P. Casey, D.T. Everett et al., Space Sci. Rev. 105, 561-599 (2003)

R.C. Wiens, M. Neugebauer, D.B. Reisenfeld, R.W. Moses Jr., J.E. Nordholt, D.S. Burnett, Space Sci. Rev. 105, 601-625 (2003)

R.C. Wiens, P. Bochsler, D.S. Burnett, R.F. Wimmer-Schweingruber, Earth Planet. Sci. Lett. 222, 697-712 (2004)

J.F. Ziegler, Nucl. Instrum. Methods Phys. Res. 219/220 1027-1036 (2004) 